\title{
P19: Analyse der Websites niedergelassener Fachärzte für Orthopädie, Fachärzte für Unfallchirurgie sowie Fachärzte für Orthopädie und Unfallchirurgie in Sachsen
}

\author{
Kristin Kocksch · Jörg Klewer
}

Online publiziert: 23. Oktober 2013

(C) Springer-Verlag Wien 2013

Einleitung/Fragestellung: Vor dem Hintergrund des zunehmenden Wettbewerbs unter medizinischen Leistungserbringern und dem steigenden Informationsbedürfnis der Patienten gewinnt für Ärzte die marketingorientierte Praxispräsentation auf einer eigenen Website zunehmend an Relevanz. In der Studie sollte daher der Internetauftritt von niedergelassenen Fachärzten für Orthopädie und/oder Unfallchirurgie in Sachsen untersucht werden.

Methodik/Stichprobe: Alle im Arztregister der Kassenärztlichen Vereinigung Sachsen gelisteten Fachärzte für Orthopädie und/oder Unfallchirurgie wurden auf Existenz und Gestaltung eines Internetauftrittes untersucht. Vorhandene Websites wurden mit Hilfe einer zuvor erstellten standardisierten Checkliste analysiert, welche unter anderem die Themenbereiche „Web Usability“, ,Barrierefreiheit“, „Dialogkomponenten“" sowie „Informationsgehalt" umfasste.

Ergebnisse: Von allen untersuchten Fachärzten $(n=349)$ besaßen 40,1\% $(n=140)$ eine eigene Praxiswebsite. Über eine Breadcrumb-Navigation verfügten $17,1 \%$ der Web- sites, eine Sitemap stellten 23,6\% der Webauftritte zur Verfügung. Die Anpassung der Schriftgröße bzw. des Kontrastes war auf 6,4\% bzw. 7,1\% der Websites möglich. Eine Online-Terminvereinbarung per E-Mail wurde auf 22,9\% der Internetauftritte bereitgestellt. Rechtlich verpflichtende Inhalte das Impressum betreffend wurden auf $45 \%$ der Internetauftritte nicht ausreichend berücksichtigt. In der Arztpraxis behandelte Krankheitsbilder wurden auf $64 \%$ der Websites genannt und bei 26,4\% hiervon direkt auf der Website oder mit Hilfe von Hyperlinks zu externen Websites näher erläutert.

Diskussion/Schlussfolgerung: Die Ergebnisse verdeutlichen, dass weiterhin ein Verbesserungsbedarf bei der Gestaltung und dem Informationsgehalt der Internetauftritte besteht. Ein Ansatzpunkt wäre die Erarbeitung eines einheitlichen Kriterienkataloges, der von den Ärzten als Entscheidungshilfe und Handlungsempfehlung genutzt werden könnte.

K. Kocksch · J. Klewer

Fakultät Gesundheits- und Pflegewissenschaften,

Westsächsische Hochschule Zwickau, Zwickau, Deutschland 